\title{
ANÁLISE DA ACURÁCIA E PRECISÃO OBTIDAS NO TRANSPORTE DE COORDENADAS COM UTILIZAÇÃO DE ESTAÇÕES FIDUCIAIS
}

\author{
Alessandro Salles Carvalho, Universidade Federal de Viçosa, ascufpr@gmail.com. \\ Moisés Ferreira Costa, Universidade Federal de Viçosa, moises@ufv.br \\ Luiz Danilo Damasceno Ferreira, Universidade Federal do Paraná, luizdanilo@ufpr.br \\ Dalto Domingues Rodrigues, Universidade Federal de Viçosa, dalto@ufv.br
}

\section{RESUMO}

O transporte de coordenadas visa a determinação posicional de pontos de interesse a partir de vértices materializados vinculados a um determinado sistema de referência, ou seja, é a determinação posicional de pontos de interesse vinculados a um sistema geodésico de referência. Deste modo, pretende-se verificar a acurácia e precisão das coordenadas de uma estação transportada denominada METE, que foi obtida por meio da utilização de 2, 3 e 6 estações de referência no ajustamento, neste trabalho designadas de estações fiduciais. Para alcançar este objetivo foram utilizadas 6 estações ativas pertencentes à Rede Brasileira de Monitoramento Contínuo (RBMC) para compor as estações fiduciais e uma estação passiva para compor a estação transportada, cujas coordenadas são homologadas pelo Instituto Brasileiro de Geografia e Estatística (IBGE). Foi realizada uma sessão de observação de 6 horas, e para o pós-processamento dos dados foi utilizado o aplicativo computacional Ashtech Solutions v. 2.7. O ajustamento das componentes vetoriais das linhas de base $(\mathrm{dx}, \mathrm{dy}, \mathrm{dz})$ foi feito por meio do método paramétrico linear com a inclusão de parâmetros ponderados ou pseudo-observações.

Palavras chave: rede GPS, ajustamento, transporte de coordenadas

\section{INTRODUÇÃO}

De acordo com Vanícek e Krakiwsky (1986), a determinação de coordenadas na superfície física da terra, no mar ou no espaço em relação a um determinado sistema de referência é chamada de posicionamento de pontos.

O posicionamento tridimensional poderá ser feito a partir de redes geodésicas estabelecidas com a utilização de observações terrestres (ângulos horizontais, verticais e distâncias) ou redes baseadas no rastreamento de satélites artificiais com a utilização do método de posicionamento relativo estático (Vanícek e Krakiwsky, 1986).

De acordo com Torge (2001), rede geodésica consiste de pontos de controle monumentados, fornecendo um sistema de referência materializado para o posicionamento terrestre em todas as escalas, quer sejam de caráter global, regional ou local. As redes globais permitem a realização de sistemas de referência definidos por convenções internacionais. Redes regionais formam a base fundamental para levantamentos nacionais ou continentais, as quais formam a base dos sistemas de geo-informação e mapeamento. Redes locais são tipicamente estabelecidas para projetos de engenharia e exploração bem como investigações geodinâmicas (Torge, 2001).

Segundo Segantine (2005), a técnica de posicionamento relativo estático com utilização da fase da onda portadora é o método mais utilizado para obter observações em uma rede geodésica GNSS. E de acordo com o IBGE (2008), a precisão desta técnica, utilizando a dupla diferença de pseudodistância e fase da portadora, permite obter uma precisão de 0,01 a $1 \mathrm{ppm}$ a um nível de confiança de $68,3 \%$. 
O objetivo do posicionamento relativo estático de acordo com Hofmann Wellenhof et al. (2008), é a determinação das coordenadas dos pontos desconhecidos em relação a ponto(s) de coordenadas conhecidas (estações de referência), ou seja, um transporte de coordenadas.

Como exemplo de aplicação tem-se a implantação de redes por meio do GNSS, onde são determinados vetores comumente denominadas de linha de base, os quais ligam os pontos constituintes da rede geodésica.

A partir do pós-processamento das observáveis obtêm-se as diferenças de coordenadas entre as estações de referência e itinerantes, bem como sua respectiva Matriz Variância Covariância (MVC). Uma vez determinado as diferenças de coordenadas entre as estações, basta somar às diferenças $(\mathrm{dx}, \mathrm{dy}, \mathrm{dz})$ as coordenadas da estação de referencia para que se obtenham as coordenadas da estação itinerante.

Porém, devido a redundância de observações e necessidade de obtenção de uma solução única, para qualquer caminho percorrido na rede, as componentes vetoriais das linhas de base (dx, dy, dz) ou diferenças de coordenadas deverão ser ajustadas utilizandose o Método dos Mínimos Quadrados (M.M.Q).

O transporte de coordenadas visa a determinação posicional de pontos de interesse a partir de vértices materializados vinculados a um determinado sistema de referência, ou seja, é a determinação posicional vinculada a um sistema geodésico de referência.

\section{OBJETIVOS}

Pretende-se analisar a acurácia e precisão obtida na determinação das coordenadas geodésicas da estação METE por meio da utilização de 2, 3 e 6 estações fiduciais.

\section{MATERIAL E MÉTODOS}

Foram utilizados dados de seis estações ativas, pertencentes a Rede Brasileira de Monitoramento Contínuo (RBMC) e de uma estação passiva a ser determinada.

A Figura 1 apresenta o croquis da distribuição espacial destas estações, as quais são denominadas de: GVAL, CEFE, RJCG, RIOD, CHPI, VARG e METE. Esta ultima não faz parte da RBMC, mas tem suas coordenadas homologadas pelo Instituto Brasileiro de Geografia e Estatística (IBGE) possibilitando a verificação da acurácia obtida no posicionamento.

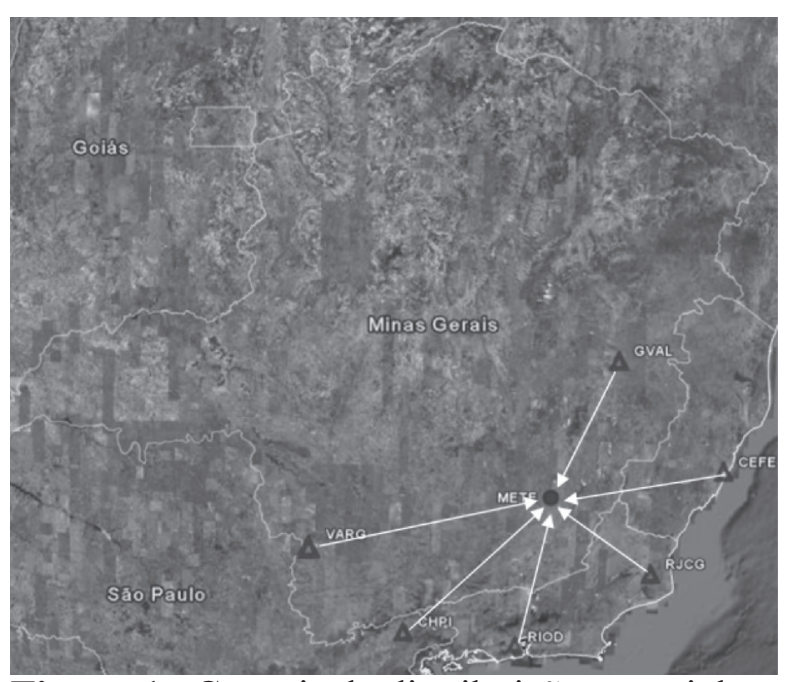

Figura 1. Croquis da distribuição espacial das estações da rede GPS.

A Figura 2 apresenta os comprimentos das Linhas de Base que compõem a rede em estudo.



Figura 2. Comprimento das Linhas de Base. 
A Figura 3 apresenta a estação METE, sendo ocupada pelo receptor geodésico descrito na Tabela 1.

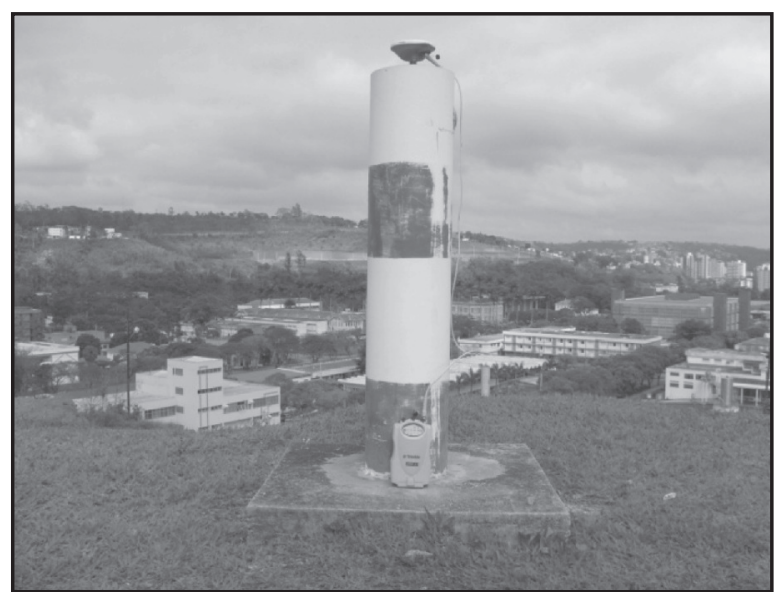

Figura 3. Rastreio da estação METE

Na Tabela 1, encontram-se os modelos de receptores e antenas instalados nas estações da Figura 1. Todos os receptores utilizados são de dupla freqüência.

Tabela 1- Receptores e Antenas instalados nas estações.

\begin{tabular}{ccc}
\hline Estação & Receptor & Antena \\
\hline GVAL & Ashtech Z - FX & ASH 700700-B \\
CEFE & NetR5 & TRM 55971.00 \\
RJCG & NetR5 & TRM 55971.00 \\
RIOD & Trimble NetRS & TRM 41249.00 \\
CHPI & Ashtech UZ -12 & ASH701945C_M \\
VARG & Ashtech Z - FX & ASH 700700-B \\
METE & Trimble 5700 & TRM 39105.00 \\
\hline
\end{tabular}

O intervalo de gravação das observações das estações ativas da RBMC é de $15 \mathrm{~s}$ e o da estação passiva de $5 \mathrm{~s}$. Vale ressaltar que o intervalo de gravação utilizado no pósprocessamento é de $15 \mathrm{~s}$, visto que, de acordo com Hofmann-Wellenhof et al. (2008), no posicionamento relativo são necessárias que as observações sejam simultâneas tanto na estação de referência quanto na estação a determinar.

A duração da sessão de observação foi de aproximadamente seis horas, sendo realizada no dia 298 do ano.
Para a realização do pós-processamento foi utilizado o aplicativo computacional Ashtech Solutions v. 2.7, utilizando-se de máscara de elevação de $15^{\circ}$ e coordenadas das estações ativas, como controle.

As coordenadas destas estações estão vinculadas ao SIRGAS 2000, época de referência $(2000,4)$, que é uma densificação do ITRF 2000 e deveriam ser convertidas em WGS 84 que é o sistema de referência do GPS, porém não há parâmetros para a realização desta transformação. De acordo com IBGE (2008), o WGS 84 (G1150) é compatível ao ITRF 2000 a um nível centimétrico. Deste modo, para fins práticos consideram-se que as coordenadas nestes dois sistemas de referência sejam compatíveis.

Após o pós-processamento foram obtidas as componentes vetoriais $(\mathrm{dx}, \mathrm{dy}, \mathrm{dz})$ das linhas de base bem como as respectivas MVC's. Realizou-se o ajustamento de observações de modo a estimar as coordenadas e precisões da estação METE.

Foram realizados seis experimentos consistindo na utilização de 2, 3 e 6 estações fiduciais. No primeiro caso, foram consideradas as estações VARG e CHPI; no segundo, as estações RIOD e RJCG, no terceiro, as estações CEFE e GVAL; no quarto caso, as estações VARG, CHPI e RIOD; no quinto caso, as estações RJCG, CEFE e GVAL e no sexto caso, todas as estações anteriores.

As linhas de base GVAL-METE, VARG-METE e CEFE-METE tiveram as ambigüidades fixadas e as linhas de base RIOD-METE, RJCG-METE e CHPI-METE tiveram solução parcial das ambigüidades.

$\mathrm{O}$ maior e menor comprimento respectivamente das linhas de base com solução fixa é de aproximadamente $280 \mathrm{~km}$ e $231 \mathrm{~km}$ e de solução parcial de 305 km e 194 $\mathrm{km}$. Na tabela 2, encontram-se as componentes vetoriais das linhas de base bem como suas respectivas variâncias. 
Tabela 2 - Componentes vetoriais das linhas de base bem como os respectivos desvios padrão.

\begin{tabular}{lccccccc}
\hline DE PARA Comprimento $(\mathrm{Km})$ & $\mathrm{DX}(\mathrm{m})$ & $\mathrm{DY}(\mathrm{m})$ & $\mathrm{DZ}(\mathrm{m})$ & $\sigma_{\mathrm{x}}(\mathrm{m})$ & $\sigma_{\mathrm{Y}}(\mathrm{m})$ & $\sigma_{\mathrm{z}}(\mathrm{m})$ \\
\hline CEFE METE & 270,044 & $-188800,960$ & $-187245,755$ & $-47082,050$ & 0,272 & 0,274 & 0,274 \\
CHPI METE & 305,876 & 209073,372 & 103275,518 & 197945,391 & 0,228 & 0,230 & 0,228 \\
RIOD METE & 232,121 & 93392,450 & $-24750,302$ & 211057,863 & 0,173 & 0,170 & 0,190 \\
RJCG METE & 194,408 & $-76666,810$ & $-145848,820$ & 103172,778 & 0,217 & 0,221 & 0,219 \\
GVAL METE & 231,490 & $-116513,305$ & $-22196,568$ & $-198795,231$ & 0,164 & 0,164 & 0,164 \\
VARG METE & 280,639 & 208169,096 & 170054,404 & 80656,120 & 0,192 & 0,179 & 0,182 \\
\hline
\end{tabular}

\section{Ajustamento Vetorial da Rede GPS}

Foi obtido como resultado do pósprocessamento das linhas de base, suas componentes vetoriais bem como suas respectivas precisões. Como existem observações (dx, dy, dz) superabundantes, utiliza-se o método dos mínimos quadrados, caso paramétrico, para estimar as coordenadas da estação METE. O método paramétrico da forma $\mathrm{La}=\mathrm{F}(\mathrm{Xa})$ foi utilizado, pois de acordo com (Leick, 1995) a cada observação é gerado uma equação de observação e é relativamente fácil sua implementação.

Nas equações de observações foram acrescentados os parâmetros ponderados ou pseudo-observações, onde foram atribuídos pesos "grandes" de forma que as coordenadas das estações de controle fossem fixadas e tidas como fiduciais.

A utilização de estações fiduciais não permitirão que as suas incertezas provenientes de um ajustamento anterior sejam propagadas na determinação dos pontos de densificação ou transportadas, visto que o peso "grande" faz com que a variável torne-se constante.

Os modelos de equação para as observações em rede GPS podem ser expressos de acordo com as equações:

$$
\begin{aligned}
& \Delta X_{i j}=X_{j}-X_{i} \\
& \Delta Y_{i j}=Y_{j}-Y_{i} \\
& \Delta Z_{i j}=Z_{j}-Z_{i} .
\end{aligned}
$$

Para os parâmetros de ligação (pontos fiduciais) têm-se as seguintes equações:

$$
\begin{aligned}
& X_{i b}=X_{i a} \\
& Y_{i b}=Y_{i a} \\
& Z_{i b}=Z_{i a}
\end{aligned}
$$

A matriz projeto, ou dos coeficientes, é constituída por elementos 1,0 e -1 com estas novas observações ou pseudo-observações ficará da seguinte forma:

$$
A=\sum_{n+1}\left[\begin{array}{l}
{ }_{n}\left[\left(\AA^{0}\right)\right]^{u} \\
{[(K)]^{u}}
\end{array}\right]^{u}
$$

Onde:

$>>\tilde{\mathbf{A}}$ : matriz dos coeficientes dos parâmetros

$(\mathbf{n} \times \mathbf{u})$;

$>>\mathbf{K}$ : matriz dos coeficientes dos pontos fiduciais (lxu),

$>>$ n: número de equações de observações (cada componente da linha de base);

>>I: número de parâmetros ponderados (estações de ligação);

> $>$ u: número de parâmetros (total de estações da rede + vértices de ligação);

K é uma matriz (lxu), devendo possuir três linhas de zeros para cada ponto de injunção e, no cruzamento dessas linhas com as três colunas referentes ao ponto de injunção correspondente, contendo uma matriz identidade de dimensão (3x3) (Vasconcellos, 2008).

O vetor das observações Lb também é acrescido das coordenadas dos parâmetros ponderados, passando a ser apresentado da seguinte forma: 


$$
\mathrm{L}_{\mathrm{b}}=\underbrace{}_{\mathrm{n}+1}\left[\begin{array}{c}
{\left[\mathrm{g} \mathrm{L}_{\mathrm{b}}^{0}\right]^{1}} \\
{\left[\hat{\mathrm{X}}_{2}\right]^{1}}
\end{array}\right]^{1}
$$

onde:

$\mathrm{\ell}_{\mathrm{b}}$ : vetor das observações (componentes das linhas de base);

$\hat{\mathrm{X}}_{2}$ : vetor dos parâmetros ponderados (coordenadas dos pontos de ligação).

A MVC das observações $\left(\sum_{L_{b}}\right)$ deve ser acrescida das variâncias dos pontos dos pontos fiduciais $\left(\sum_{\hat{x}_{2}}\right)$, preenchendo os demais elementos com zero. A matriz dos pesos deste modo assumirá a seguinte forma:

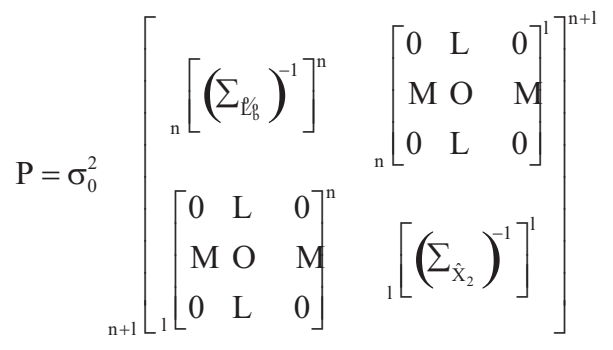

A matriz dos pesos utilizado neste trabalho teve a forma diagonal, pois estão presentes somente as variâncias das componentes vetoriais das linhas de base.

Como resultado do ajustamento, notase que as coordenadas dos pontos fiduciais aparecem nos vetores das observações e parâmetros ajustados, visto que estes foram considerados parâmetros observados. O mesmo acontece com as MVC's das observações e dos parâmetros ajustados.

Os parâmetros ajustados $\mathrm{X}_{\mathrm{a}}^{\circ}$ são determinados pela seguinte equação:

$$
\mathrm{X}_{\mathrm{a}}^{0}=\mathrm{N}^{-1} \mathrm{U}
$$

onde:

$\mathrm{P}$ : matriz dos pesos referentes às observações e aos parâmetros ponderados;

$\mathrm{N}=\mathrm{A}^{\mathrm{T}} \mathrm{PA}$ : matriz dos coeficientes das equações normais;
$\mathrm{U}=\mathrm{A}^{\mathrm{T}} \mathrm{PL}_{\mathrm{b}}$ : termo independente das equações normais.

O vetor dos parâmetros ajustados é dado por:

$$
\hat{\mathrm{X}}_{\mathrm{a}}={ }_{\mathrm{n}+1}\left[\begin{array}{c}
{\left[\hat{\mathrm{X}}_{1}^{\mathrm{a}}\right]^{1}} \\
{\left[\hat{\mathrm{X}}_{2}^{\mathrm{a}}\right]^{1}}
\end{array}\right]^{1}
$$

A estimativa de precisão dos parâmetros ajustados é dada por:

$$
\sum_{\hat{\mathrm{X}}_{\mathrm{a}}}=\hat{\sigma}_{0}^{2}\left(\mathrm{~A}^{\mathrm{T}} \mathrm{PA}\right)^{-1}
$$

onde:

$\hat{\sigma}_{0}^{2}$ : fator de variância a posteriori calculado pela equação (16).

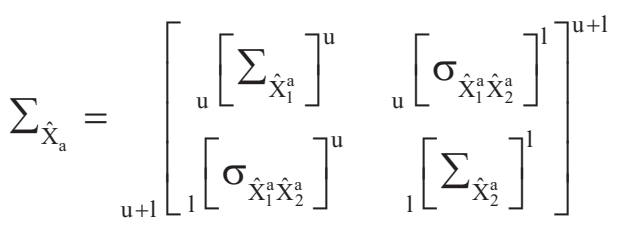

onde:

$\hat{X}_{1}^{a}$ : parâmetros ajustados pertencentes à rede GPS;

$\hat{X}_{2}^{a}$ : parâmetros ajustados referentes as estações fiduciais. dado por:

O vetor das observações ajustadas é

$$
\hat{\mathrm{L}}_{\mathrm{a}}=\mathrm{A} \hat{\mathrm{X}}_{\mathrm{a}}
$$

e a MVC das observações é dado por:

$$
\sum_{\hat{\mathrm{La}}}=\hat{\sigma}_{0}^{2} \mathrm{AN}^{-1} \mathrm{~A}^{\mathrm{T}} \text {. }
$$

As observações ajustadas, bem como suas estimativas de precisão são dadas pelas equações (12) e (13), respectivamente. 
$\mathrm{L}_{\mathrm{a}}=\left[\begin{array}{c}\left.\left[\mathrm{n}_{\mathrm{a}}\right]^{1}\right]^{1} \\ {\left[\mathrm{X}_{2}^{\mathrm{a}}\right]^{1}}\end{array}\right]^{1}$,

e,

$$
\sum_{L_{a}}=\left[\begin{array}{cc}
{ }_{n}\left[\sum_{L_{a}}\right]^{n} & { }_{n}\left[\sigma_{L_{a}, X_{2}^{a}}\right]^{1} \\
{\left[\sigma_{L_{a}, X_{2}^{a}}\right]^{n}} & {\left[\sum_{X_{2}^{a}}\right]^{1}}
\end{array}\right]^{n+1}
$$

O vetor dos resíduos é determinado pela equação:

$$
\mathrm{V}=\mathrm{AX} \hat{\mathrm{a}}_{\mathrm{a}}-\mathrm{Lb},
$$

o qual é dado na forma:

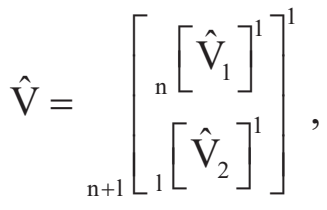

onde:

$\hat{\mathrm{V}}_{1}$ : vetor dos resíduos referente às observações (componentes das linhas de base);

$\hat{\mathrm{V}}_{2}$ : vetor dos resíduos referentes aos parâmetros ponderados.

O fator de variância da unidade de peso aposteriori $\hat{\sigma}_{0}^{2}$ é estimado por:

$$
\hat{\sigma}_{0}^{2}=\frac{\mathrm{V}^{\mathrm{T}} \mathrm{PV}}{\mathrm{n}+1-\mathrm{u}}
$$

O numerador é a soma acumulada dos $V^{\top} P V$ das observações e dos pontos fiduciais incluídos como observações, o denominador é o número de observações superabundantes, ou seja, o grau de liberdade (Gemael, 1994).

\section{Cálculos do comprimento do arco de meridiano e paralelo}

De posse das coordenadas cartesianas obtidas no processo de ajustamento, foram obtidas as coordenadas geodésicas curvilíneas da estação estimada por meio do programa
ProGriD v.1, desenvolvido no âmbito do Projeto de Infra-estrutura Geoespacial Nacional (PIGN).

Deste modo, pode-se avaliar a acurácia obtida na latitude $(\varphi)$, longitude $(\lambda)$ e altitude geométrica $(h)$. Para a conversão dos valores de acurácia dados em segundos de arco para comprimento de arco, foram utilizados a seguintes equações (Torge, 2001).

$$
\begin{aligned}
& \mathrm{dG}=\mathrm{Md} \varphi \\
& \mathrm{dL}=\mathrm{N} \cos \varphi \mathrm{d} \lambda \\
& \mathrm{d} \varphi=\varphi_{2}-\varphi_{1} \\
& \mathrm{~d} \lambda=\lambda_{2}-\lambda_{1} \\
& \mathrm{M}=\frac{\mathrm{a}\left(1-\mathrm{e}^{2}\right)}{\left(1-\mathrm{e}^{2} \operatorname{sen}^{2} \varphi\right)^{3 / 2}} \\
& \mathrm{~N}=\frac{\mathrm{a}}{\left(1-\mathrm{e}^{2} \operatorname{sen}^{2} \varphi\right)^{1 / 2}}
\end{aligned}
$$

onde:

$\mathrm{dG}$ : comprimento do arco ao longo do meridiano;

$\mathrm{dL}$ : comprimento do arco ao longo do paralelo.

$\mathrm{d} \varphi$ : diferença de latitude, dado em radianos.

$\mathrm{d} \lambda$ : diferença em longitude, dado em radianos;

$\mathrm{e}^{2}$ : primeira excentricidade ao quadrado;

$\varphi$ : latitude do ponto;

$\lambda$ : longitude do ponto;

a : semi-eixo maior do elipsóide de revolução adotado.

M : raio de curvatura da seção meridiana;

$\mathrm{N}$ : raio de curvatura da seção primeiro vertical.

O elipsóide utilizado para o cálculo dos raios de curvatura da seção meridiana e seção primeiro vertical foi o elipsóide do sistema de referência geodésico de 1980 (Geodetic Reference System 1980 (GRS 80)), cujos parâmetros são: semi-eixo maior (a) igual a $\mathrm{a}=6378137$ e achatamento igual a $\mathrm{f}=1 / 298,257222101$. Este foi o elipsóide escolhido, visto que as coordenadas das estações fiduciais estão em SIRGAS 2000 e tem como modelo matemático este elipsóide. 
estações fiduciais estão em SIRGAS 2000 e tem como modelo matemático este elipsóide.

\section{RESULTADOS E DISCUSSÕES}

Foi realizado o ajustamento do primeiro caso, no qual foram consideradas as coordenadas cartesianas das estações VARG e CHPI como observações ponderadas. O número de graus de liberdade foi igual a 3 . O fator de variância a posteriori foi de $0,095 \mathrm{~m}^{2}$ e a estatística qui-quadrado calculado foi de 0,284 . Aplicando o teste global do modelo ao nível de 5\% significância, verifica-se que a hipótese nula é aceita, ou seja, os fatores de variância a priori e a posteriori são estatisticamente iguais ao nível de $95 \%$ de confiança.

As coordenadas cartesianas e precisões da estação METE podem ser encontradas na tabela 3 .

Tabela 3 - Coordenadas cartesianas e respectivas precisões da estação METE.

\begin{tabular}{ccccccc}
\hline Estação & $\mathrm{X}(\mathrm{m})$ & $\mathrm{Y}(\mathrm{m})$ & $\mathrm{Z}(\mathrm{m})$ & $\sigma_{\mathrm{X}}(\mathrm{m})$ & $\sigma_{\mathrm{Y}}(\mathrm{m})$ & $\sigma_{\mathrm{Z}}(\mathrm{m})$ \\
\hline METE & 4373687,326 & $-4059181,358$ & $-2247083,510$ & 0,045 & 0,044 & 0,044 \\
\hline
\end{tabular}

Foi realizado o ajustamento do segundo caso, considerando as coordenadas das estações RIOD e RJCG como observações ponderadas. O número de graus de liberdade foi igual a 3 . O fator de variância a posteriori foi de $0,133 \mathrm{~m}^{2}$ e a estatística qui-quadrado calculado foi de 0,400 .

Aplicando o teste global do modelo ao nível de 5\% significância, verifica-se que a hipótese nula é aceita, ou seja, os fatores de variância a priori e a posteriori são estatisticamente iguais ao nível de $95 \%$ de confiança.

As coordenadas cartesianas e precisões da estação METE podem ser encontradas na tabela 4.

Tabela 4 - Coordenadas cartesianas e respectivas precisões da estação METE

\begin{tabular}{ccccccc}
\hline Estação & $\mathrm{X}(\mathrm{m})$ & $\mathrm{Y}(\mathrm{m})$ & $\mathrm{Z}(\mathrm{m})$ & $\sigma_{\mathrm{X}}(\mathrm{m})$ & $\sigma_{\mathrm{Y}}(\mathrm{m})$ & $\sigma_{\mathrm{Z}}(\mathrm{m})$ \\
\hline METE & 4373687,377 & $-4059181,559$ & $-2247083,558$ & 0,049 & 0,049 & 0,052 \\
\hline
\end{tabular}

Foi realizado o ajustamento do terceiro caso, considerando as coordenadas das estações CEFE e GVAL como observações ponderadas. O número de graus de liberdade foi igual a 3. O fator de variância a posteriori foi de $0,013 \mathrm{~m}^{2}$ e a estatística qui-quadrado calculado foi de 0,039 .
Aplicando o teste global do modelo ao nível de 5\% significância, verifica-se que a hipótese nula não é aceita, ou seja, os fatores de variância a priori e a posteriori são estatisticamente diferentes ao nível de $95 \%$ de confiança.

As coordenadas cartesianas e precisões da estação METE podem ser encontradas na tabela 5 .

Tabela 5 - Coordenadas cartesianas e respectivas precisões da estação METE

\begin{tabular}{ccccccc}
\hline Estação & $\mathrm{X}(\mathrm{m})$ & $\mathrm{Y}(\mathrm{m})$ & $\mathrm{Z}(\mathrm{m})$ & $\sigma_{\mathrm{X}}(\mathrm{m})$ & $\sigma_{\mathrm{Y}}(\mathrm{m})$ & $\sigma_{\mathrm{Z}}(\mathrm{m})$ \\
\hline METE & 4373687,507 & $-4059181,515$ & $-2247083,635$ & 0,016 & 0,016 & 0,016 \\
\hline
\end{tabular}


Das tabelas 3, 4 e 5 pode-se observar que as melhores precisões alcançadas na determinação das coordenadas da estação METE foi obtida no terceiro caso, embora não tenha passado no teste global do modelo a 5\% de significância.

Como para a determinação das precisões dos parâmetros estimados é necessário a utilização do fator de variância a posteriori e como este, no terceiro caso, foi menor que os outros dois casos, pode ter feito com que os valores das precisões dos parâmetros estimados fossem menores. Foi realizado o ajustamento do quarto caso, considerando as coordenadas das estações
VARG, CHPI e RIOD como observações ponderadas. O número de graus de liberdade foi igual a 6 . O fator de variância a posteriori foi de $0,148 \mathrm{~m} 2$ e a estatística qui-quadrado calculado foi de 0,889 .

Aplicando o teste global do modelo ao nível de 5\% significância, verifica-se que a hipótese nula foi aceita, ou seja, os fatores de variância a priori e a posteriori são estatisticamente iguais ao nível de $95 \%$ de confiança.

As coordenadas cartesianas e precisões da estação METE podem ser encontradas na Tabela 6.

Tabelas 6 - Coordenadas cartesianas e respectivas precisões da estação METE

\begin{tabular}{ccccccc}
\hline Estação & $\mathrm{X}(\mathrm{m})$ & $\mathrm{Y}(\mathrm{m})$ & $\mathrm{Z}(\mathrm{m})$ & $\sigma_{\mathrm{X}}(\mathrm{m})$ & $\sigma_{\mathrm{Y}}(\mathrm{m})$ & $\sigma_{\mathrm{Z}}(\mathrm{m})$ \\
\hline METE & 4373687,327 & $-4059181,427$ & $-2247083,507$ & 0,043 & 0,042 & 0,041 \\
\hline
\end{tabular}

Foi realizado o ajustamento do quinto caso, considerando as coordenadas das estações RJCG, CEFE e GVAL como observações ponderadas. O número de graus de liberdade foi igual a 6 . O fator de variância a posteriori foi de $0,038 \mathrm{~m}^{2}$ e a estatística quiquadrado calculado foi de 0,226. Aplicando o teste global do modelo ao nível de 5\% significância, verifica-se que a hipótese nula foi aceita, ou seja, os fatores de variância a priori e a posteriori são estatisticamente iguais ao nível de $95 \%$ de confiança.

As coordenadas cartesianas e precisões da estação METE podem ser encontradas na Tabela 7.

Tabelas 7 - Coordenadas cartesianas e respectivas precisões da estação METE

\begin{tabular}{ccccccc}
\hline Estação & $\mathrm{X}(\mathrm{m})$ & $\mathrm{Y}(\mathrm{m})$ & $\mathrm{Z}(\mathrm{m})$ & $\sigma_{\mathrm{X}}(\mathrm{m})$ & $\sigma_{\mathrm{Y}}(\mathrm{m})$ & $\sigma_{\mathrm{Z}}(\mathrm{m})$ \\
\hline METE & 4373687,491 & $-4059181,543$ & $-2247083,628$ & 0,023 & 0,023 & 0,023 \\
\hline
\end{tabular}

Como ultimo experimento, sexto caso, será verificado a acurácia obtida com a utilização das estações VARG, CHPI, RIOD, RJCG, CEFE e GVAL como fiduciais na realização do ajustamento. O número de graus de liberdade foi igual a 15 . O fator de variância a posteriori foi de $0,208 \mathrm{~m}^{2}$ e a estatística quiquadrado calculado foi de 3,125 .
Aplicando o teste global do modelo ao nível de 5\% significância, verifica-se que a hipótese nula foi aceita, ou seja, os fatores de variância a priori e a posteriori são estatisticamente iguais ao nível de $95 \%$ de confiança.

As coordenadas cartesianas e precisões da estação METE podem ser encontradas na Tabela 8. 
Tabelas 8 - Coordenadas cartesianas e respectivas precisões da estação METE

\begin{tabular}{|c|c|c|c|c|c|c|}
\hline Estação & $\mathrm{X}(\mathrm{m})$ & $\mathrm{Y}(\mathrm{m})$ & $\mathrm{Z}(\mathrm{m})$ & $\sigma_{\mathrm{X}}(\mathrm{m})$ & $\sigma_{\mathrm{Y}}(\mathrm{m})$ & $\sigma_{\mathrm{Z}}(\mathrm{m})$ \\
\hline METE & 4373687,405 & $-4059181,480$ & $-2247083,568$ & 0,037 & 0,037 & 0,037 \\
\hline
\end{tabular}

A Figura 4 apresenta a acurácia obtida na determinação das coordenadas cartesianas da estação METE sob diferentes estratégias.

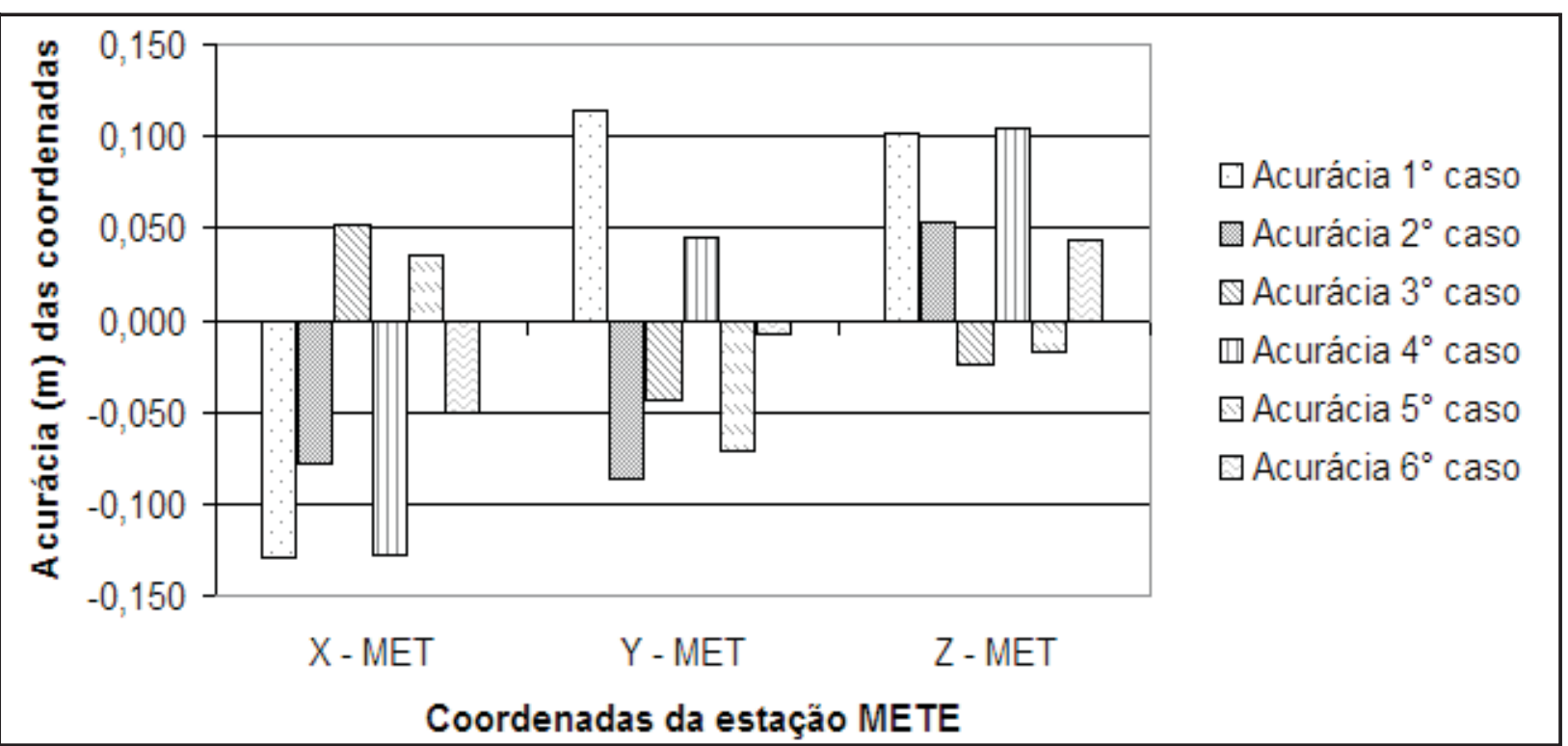

Figura 4. Acurácia obtida na determinação da estação METE usando coordenadas cartesianas.

A Figura 5 apresenta as medidas de acurácia obtidas na determinação das coordenadas geodésicas curvilíneas da estação METE nos seis diferentes casos.

Para a obtenção dos valores de acurácia em medidas lineares as diferenças angulares para a latitude foram multiplicadas por $30,75 \mathrm{~m}$ e em longitude por $28,93 \mathrm{~m}$. Estes valores podem ser obtidos por meio das equações (17) e (18).

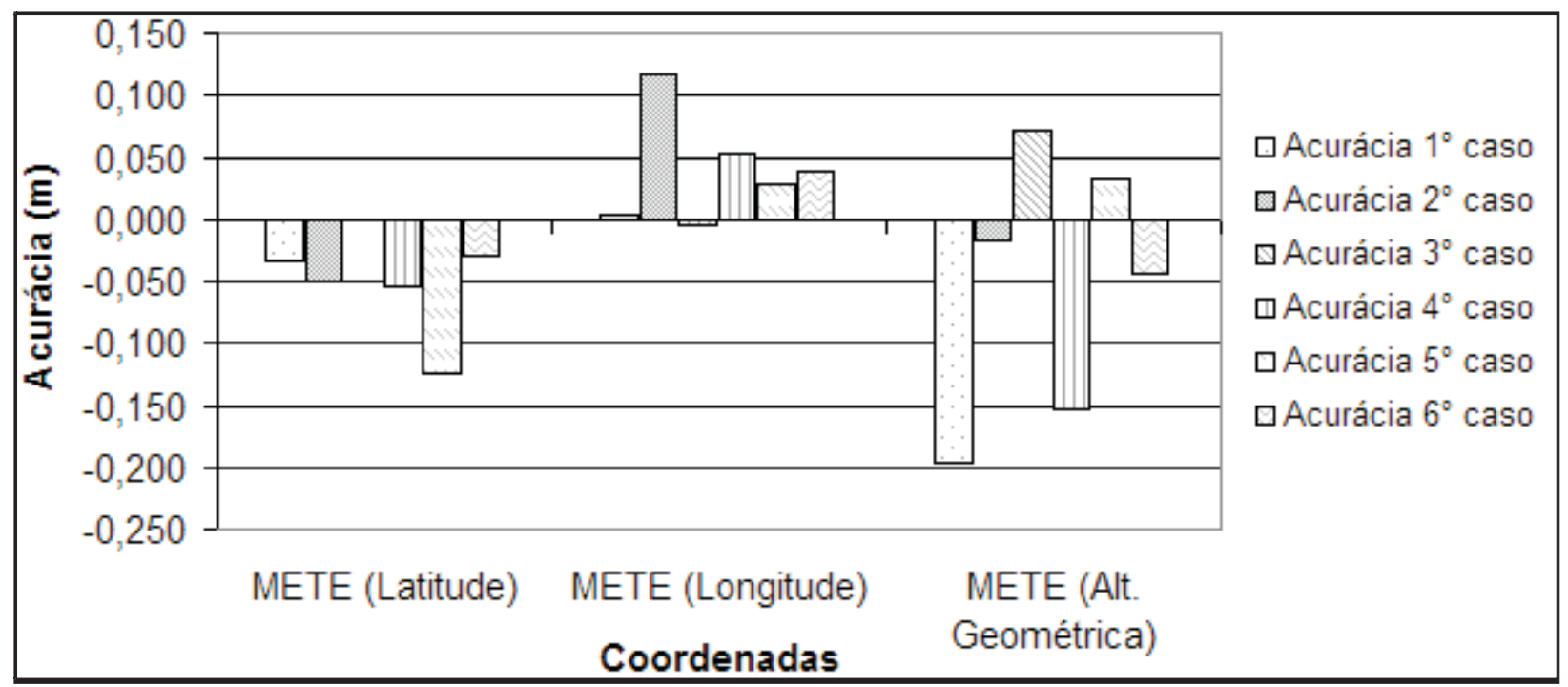

Figura 5. Acurácia obtida na determinação da estação METE usando coordenadas elipsóidicas. 
Das Figuras 4 e 5 percebe-se que a análise de acurácia posicional deve ser feito em termos de coordenadas geodésicas (Latitude, Longitude e Altitude Geométrica), haja vista que para o cálculos destas, são necessárias a utilização conjunta das coordenadas cartesia- nas. A Figura 6 apresenta a acurácia posicional plana e altimétrica obtida na estação METE, nos diferentes casos. A acurácia posicional no plano foi calculada aplicando o teorema de Pitágoras nas medidas de acurácia Latitude e Longitude.

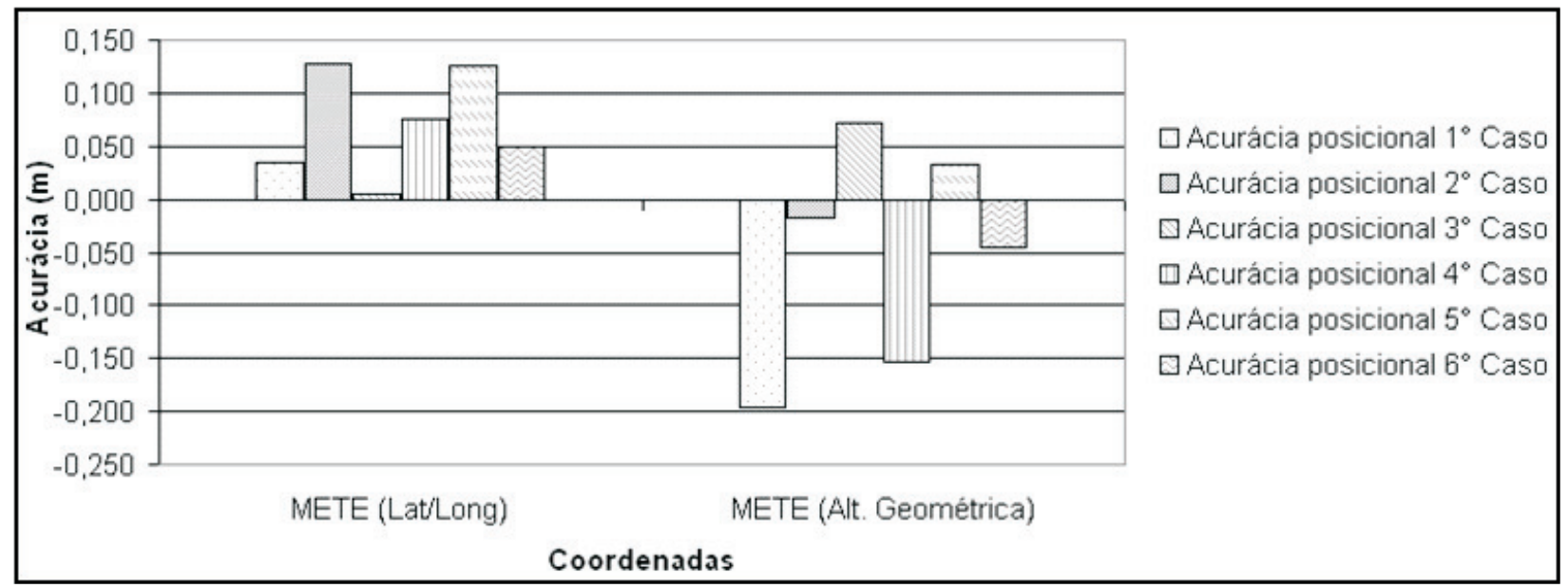

Figura 6. Acurácia plana e altimétrica obtida na determinação da estação METE.

Na Figura 6, percebe-se que a acurácia difere em cada caso, sendo que foi obtida uma melhor acurácia posicional plana no terceiro caso, e altimétrica no segundo caso.
Na Figura 7, observam-se as mediadas de acurácia posicional tridimensional obtida na determinação das coordenadas da estação METE. Destas, percebe-se que a melhor estimativa foi realizada no sexto caso.

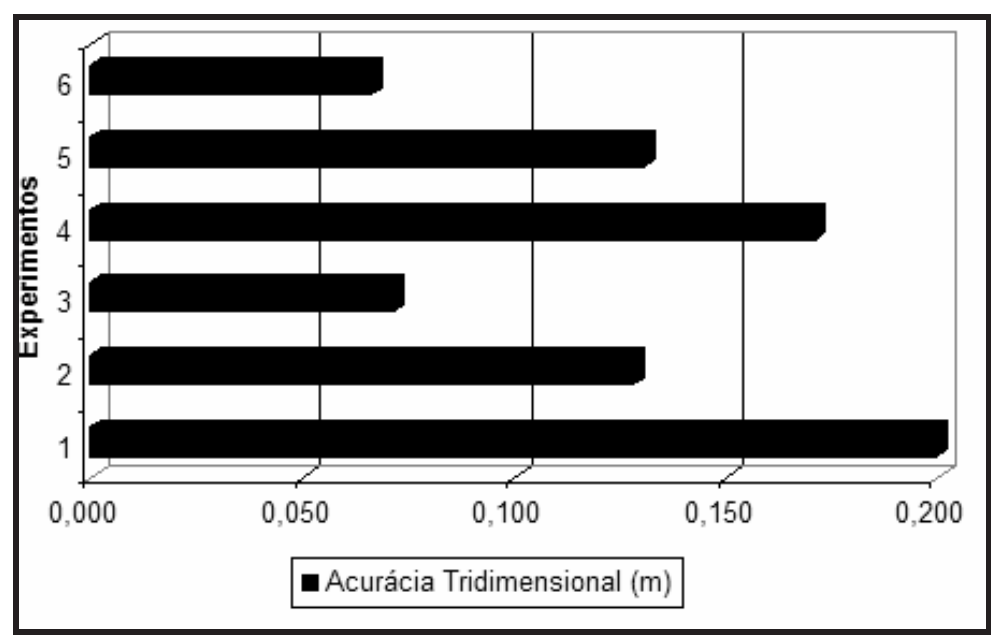

Figura 7 - Acurácia tridimensional da estação METE.

Da Figura 7, tem-se que dos casos em que foram consideradas somente duas estações fiduciais, a terceira situação proporcionou um melhor nível de acurácia. Nos casos em que foram consideradas 3 estações fiduciais, a $5^{\mathrm{a}}$ situação apresentou melhores resultados. De modo geral, a situação que apresentou melhor acurácia tridimensional foi obtida no caso em que foram consideradas todas as estações ativas como fiduciais. 


\section{CONCLUSÃO}

Pode-se verificar que o número de estações, bem como a escolha combinada destas, proporciona níveis de precisão e acurácia distintos, sinalizando para um processo de otimização, haja vista que poderão ser realizados testes de modo que se possam escolher estações que proporcionem melhores valores posicionais estimados.

Embora o terceiro caso não tenha obtido um bom desempenho de acordo com o teste global do modelo ao nível de 5\% de significância, foram obtidas neste, os melhores níveis de precisão e acurácia comparativamente aos casos 1 e 2 em que foram utilizadas 2 estações fiduciais.

Como era esperada, a acurácia posicional plana foi melhor do que a acurácia vertical. Nota-se que o aplicativo computacional utilizado não fornece a opção de o usuário entrar com modelo troposférico, nem ionosférico o que de certo modo dificulta a resolução das ambigüidades e conseqüentemente uma determinação das componentes vetoriais das linhas de base menos acuradas.

Verifica-se que poderá ser obtida uma boa precisão na estimativa das coordenadas, sem, contudo, obter uma boa medida de acurácia. Deste modo, obter boa precisão não implica em boa acurácia e vice-versa.

Como no sexto caso o número de observações foi maior que nos demais e conseqüentemente o número de graus de liberdade, era de se esperar uma boa acurácia posicional tridimensional e confiabilidade, o que ocorreu.

\section{REFERÊNCIAS BIBLIOGRÁFICAS}

DALMOLIN, Q. Ajustamento por mínimos quadrados. 2. ed. Curitiba: Universidade Federal do Paraná. Curso de pós-graduação em Ciências Geodésicas. Editora UFPR, 2004.
GEMAEL, C. Introdução ao ajustamento de observações: aplicações geodésicas. Curitiba: Editora UFPR, 1994.

HOFMANN-WELLENHOF, B. et al. GNSS Global Navigation Satellite Systems: GPS, GLONASS, GALILEO \& more. Austria: Springer Wien New York, 2008.

IBGE - Fundação Instituto Brasileiro de Geografia e Estatística. Recomendações para Levantamentos Relativos Estáticos - GPS. Rio de Janeiro, 2008.

LEICK, A. GPS satellite surveying. 2nd. ed. New York: John Wiley and Sons, 1995.

SEGANTINE, P. C. GPS: sistema de posicionamento global. São Carlos: EESC/USP, 2005.

TORGE, W. Geodesy. 3rd ed. Berlin, New York: de Gruyter, 2001.

VANÍCEK, P., KRAKIWSKY, E.J. Geodesy: the Concepts. 2nd ed. Netherlands. North Holland Publishing, 1986.

VASCONCELLOS, J. C. P. Metodologia para realização do ajustamento de redes geodésicas GPS de densificação ou extensão. Revista Brasileira de Cartografia, n. 60/01. 20008 . D i s p o n íve 1 e m: < http://www.rbc.ufrj.br/_2008/60_1_06.htm>. Acesso em: 28 fev. 2009. 